\title{
Belphégor
}

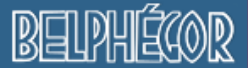

Littérature populaire et culture médiatique

18-2 | 2020

Roman Populaire Espagnol - Roman historique, 1900-1950

\section{Boillat Alain, Philippe Gilles, L'Adaptation. Des livres aux scénarios}

Irène Langlet

\section{(2) OpenEdition \\ Journals}

Electronic version

URL: http://journals.openedition.org/belphegor/3376

DOI: $10.4000 /$ belphegor.3376

ISSN: 1499-7185

Publisher

LPCM

Electronic reference

Irène Langlet, "Boillat Alain, Philippe Gilles, L'Adaptation. Des livres aux scénarios", Belphégor [Online],

18-2 | 2020, Online since 16 December 2020, connection on 28 April 2021. URL: http://

journals.openedition.org/belphegor/3376 ; DOI: https://doi.org/10.4000/belphegor.3376

This text was automatically generated on 28 April 2021.

\section{(c)}

Belphégor est mis à disposition selon les termes de la Licence Creative Commons Attribution - Pas d'Utilisation Commerciale - Pas de Modification 4.0 International. 


\section{Boillat Alain, Philippe Gilles, L'Adaptation. Des livres aux scénarios}

Irène Langlet

\section{REFERENCES}

Boillat Alain, Philippe Gilles, L'Adaptation. Des livres aux scénarios, Bruxelles : Les Impressions nouvelles, 2018, 352 p., bibliographie, index

\section{L'ADAPTATION}

Des livres aux scénarios

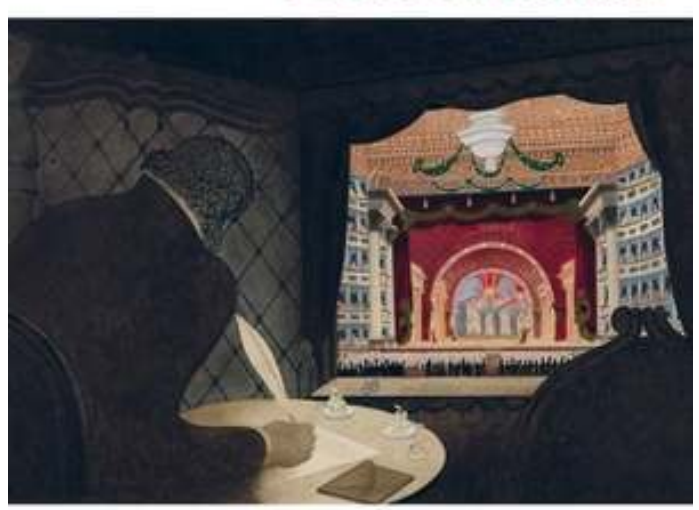

Ouvrage sous la direction d'Alain Boillat et Gilles Philippe 
1 Considérée comme l'une des plus importantes institutions de conservation d'archives du cinéma au monde, la Cinémathèque Suisse, fondée en 1948, a formalisé dans un partenariat officiel en 2010 les relations qu'elle entretenait de longue date avec l'université de Lausanne, et notamment sa section d'histoire et d'esthétique du cinéma fondée en 1990. Dans le cadre de cette coopération, d'ambitieux projets de recherche ont été menés, soutenus par le Fonds national suisse de la recherche scientifique (FNS) : sur le rôle du cinéma dans l'aviation, la marque d'appareils Bolex, ou encore le vedettariat au prisme des théories du genre (gender). C'est de l'un de ces projets, «Discours du scénario : étude historique et génétique des adaptations cinématographiques de Stendhal» (dirigé par Alain Boillat, Gilles Philippe, Vincent Verselle de 2013 à 2017) que sont issus deux collectifs publiés aux Impressions Nouvelles: L'Adaptation. Des livres aux scénarios, publié en 2018 (sous la direction d'A. Boillat et G. Philippe), et Films à lire. Des scénarios et des livres, publié en 2019 sous la direction de Mireille Brangé et Jean-Louis Jeannelle. Les collectifs rassemblent les contributions à deux colloques «jumeaux" qui se sont tenus en octobre 2016, l'un à l'université de Lausanne, l'autre dans les universités de Rouen et Paris-13 Villetaneuse. Chacun des événements a donné lieu à un quadrillage minutieux de la question du scénario, pièce déterminante de la fabrique et de la culture du cinéma, en suivant deux voies : une voie génétique, où le scénario est le pivot d'une enquête sur la genèse des films, même quand celle-ci a recours à d'autres types de documents (correspondances, croquis, etc.) ; une voie plus large où le scénario, toujours avec les autres documents qui forment avec lui l'écosystème de la création et de la production, est pris et étudié comme le support d'une expérience de lecture, elle-même dimension originale mais effective de l'expérience cinématographique, en tant que culture globale. On rendra compte ici du premier volume, qui emprunte ses méthodes à la critique génétique - la revue Genesis a d'ailleurs publié dès 2015 une première étude ${ }^{1}$, issue du même projet, qui prend appui sur le fonds principalement arpenté : le fonds Claude Autant-Lara, confié à la Cinémathèque Suisse dans les années 1980 dans un contexte sur lequel il sera peut-être bon de revenir.

2 L'introduction de A. Boillat et G. Philippe explique que le titre : «L'adaptation » doit se comprendre de deux façons : passage de l'écrit à l'écran, comme dans le sens le plus courant; mais aussi, de façon plus précise, passage d'un texte à un autre texte, voire à plusieurs autres textes, la notion-même de "scénario " ne s'étant fixée que petit à petit, entre les années 1920 et les années 1950. Cette précision n'est pas que d'échelle: elle engage toute la complexité des différents processus d'écriture requis par ces productions trop souvent réduites - et, comme telles, opposées aux productions littéraires - à des «images qui bougent » ou au «tout-image» (pour le dire avec les mots de Marc Cerisuelo). À rebours d'une pratique consistant à cliver nettement les spécificités de chaque médium, les chercheurs ici ont détaillé les relations complexes tissées entre tous les membres de cette création collective qu'est un film, et les pratiques proprement littéraires des scénaristes. Il ne s'agit donc pas seulement de rapporter l'écriture aux techniques du film, mais de s'intéresser à l'écriture du scénario pour lui-même, et aux effets de sens qu'il prend dans la tension entre l'œuvre littéraire, dont il est un "après-texte », et le film, dont il est un "avant-texte ». L'ouvrage se place donc plus dans le sillage d'approches issues du champ littéraire, de la revue Genesis (dossier sur le cinéma, en 2007) aux travaux de Jean-Louis Jeannelle (Films sans images. Une histoire des scénarios non réalisés de la Condition Humaine, Seuil, 2015). 
3 La méthode génétique se justifie essentiellement par le projet d'exploiter le fonds Autant-Lara de la Cinémathèque Suisse. Les directeurs du projet la rapportent toutefois aussi à la période considérée, celle de la "Qualité française» (1946-1958), dont ils rappellent qu'elle a engagé le redressement de la production en France après la guerre (et du coup une compétition avec les productions américaines) et ensuite constitué la cible de critiques appelées à un brillant avenir : celles des auteurs de la Nouvelle Vague, emmenés par Truffaut dans sa célèbre charge sur «Une certaine tendance du cinéma français » dans les Cahiers du cinéma (janvier 1954). Au niveau historique, académismes et mouvements de rupture sont saisis ensemble, "Qualité française » et "style NRF » d'un côté, Nouvelle Vague et Nouveau Roman de l'autre; au niveau de l'écriture, une esthétique de l'adaptation se repère dans la manière de concevoir les scénarios, même quand ils n'adaptent pas d'œuvre à proprement parler. Valorisation du scénario pour lui-même et relecture de la "Qualité française» sont ainsi associées dans une entreprise génétique à plusieurs niveaux, dont le métier de scénariste lui-même n'est pas le moindre, pris entre le dialoguiste et l'adaptateur.

4 Le premier des onze chapitres du livre, signé par Laurent Le Forestier, développe une passionnante histoire des premiers "scénarios » (les guillemets importent puisque ces textes ne sont pas encore désignés ainsi, et ne sont désormais archivés comme tels qu'au prix d'un biais téléologique), qui fait prendre conscience de la diversité formidable des types d'écrits qui ont accompagné la production des années 1920-1930. Le fil conducteur de son propos est la professionnalisation progressive des métiers, telle qu'elle se lit dans l'histoire culturelle, syndicale et industrielle et dans les règles et pratiques d'écriture et de présentation des notes destinées à guider le metteur en scène. Canevas, découpage, récit écrit, scénario technique, plan de tournage : ces divers documents témoignent de la spécialisation progressive de l'adaptateur et du découpeur vis-à-vis des "littérateurs ", dialoguistes et écrivains. Aux uns les mots, aux autres les images. L'article est particulièrement éclairant en ce qu'il parvient à articuler le processus de transformation d'un texte en document technique avec les conditions industrielles et sociales liées de la production - par exemple concernant la reconnaissance des différentes tâches, que ce soit au générique ou en termes de pourcentage des recettes du film. Le Forestier fait ainsi l'hypothèse que le peu d'intérêt des écrivains à l'écriture de scénarios s'explique par le peu de reconnaissance pécunière qu'ils peuvent en attendre (paiement au forfait), par contraste avec ce qui est alors en vigueur au théâtre (paiement en pourcentage des recettes). La stabilisation du "scénario » comme écriture du plan de tournage, par opposition au "canevas » plus littéraire, se fait sous l'influence de la méthode américaine d'organisation de la production, dans les années 1930. Une autre opposition est également utilisée, entre "premier découpage », tenant à l'adaptation d'une œuvre en scènes principales, et "second découpage ", plus technique, qui prévoit le plan de tournage et les séquences précises. "Scénario » et "adaptation » peuvent ainsi coexister au générique; la présentation en deux colonnes se généralise dans les années 1930, ainsi qu'un processus en trois temps synthétisant les logiques et dynamiques de cette production collective : canevas littéraire, adaptation, découpage.

5 Trois articles s'attachent ensuite aux rapports de Gide et Bernanos au cinéma, en se rapportant notablement aux scénarios d'Aurenche et Bost. Dans le premier, Pierre Mathieu soutient la thèse d'un Gide moins maladroit à l'adaptation de ses œuvres que l'histoire peut le laisser penser. Celles-ci sont étudiées du point de vue des scénarios 
qu'il en a conçus, seul pour la Symphonie pastorale, avec P. Herbart pour Isabelle - dans le premier cas, le scénario est abandonné pour celui d'Aurenche et Bost jugé plus professionnel; dans le second, il est refusé par Cocteau. Gilles Philippe, dans l'article suivant, étudie un cas où le professionnalisme des deux scénaristes est resté insuffisant : Bernanos refusa leur proposition pour l'adaptation du Journal d'un curé de campagne. G. Philippe, en suivant une enquête génétique parfois acrobatique, par exemple des documents qui existent à coup sûr mais qui n'ont pas pu être consultés, suggère qu'Aurenche et Bost auraient imprimé une patte un peu trop "gidienne » au roman, par des sous-entendus insupportables à Bernanos mais surtout en omettant notamment d'adapter le dispositif du journal intime, et donc en perdant l'économie générale d'équilibre des passions et des grâces que toute l'œuvre de l'écrivain s'attache à développer (par exemple, et justement dans le domaine du cinéma et à la même période, dans le « Dialogue des Carmélites ${ }^{2}$ » qu'il élabore pour l'adaptation au cinéma d'une pièce de Gertrud von Le Fort en 1947). La reconstitution minutieuse du dossier génétique s'articule de façon très convaincante avec le suivi poétique des textes romanesque et scénaristique. C'est un peu moins le cas de l'article suivant, consacré au même ensemble Journal et scénario d'Aurenche et Bost. La lecture strictement textuelle et formaliste de Vincent Verselle confronte les logiques diaristes du roman et les logiques audiovisuelles qui sous-tendent le scénario en supposant que les "points d'entrée au monde » de chaque texte sont contradictoires.

Cinq études sont ensuite proposées sur l'œuvre de Claude Autant-Lara, dont le fonds déposé à la Cinémathèque Suisse se voit ainsi exploré en profondeur. Sarah Leahy met l'accent, dans l'étude du Diable au corps, sur les logiques de circulation et de compétition internationale dans le contexte de l'immédiat après-guerre. Le dossier du film est lu dans toute son hétérogénéité (scénarios, revues de presse, contrats, correspondance) pour en faire l'exemple d'une contrsuction symbolique nationale destinée à lutter contre le flot des productions américaines. La «Qualité française » désignerait, pour ses défenseurs - et en particulier la CGT, dont Autant-Lara est adhérent à ce moment-là non seulement des films et des sujets mais aussi une manière de les produire. Hormis un adjectif étrangement orienté («syndicalisés », p. 139, où on suppose qu'il faut lire "syndiqués») et une illustration un peu obscure (fig. 1: en quoi la pose de Gérard Philipe devant un mur où s'étale le mot "DÉFENSE » illustre-t-elle la lâcheté du patronat américain devant la censure?), l'article démontre de façon parfaitement convaincante et, de surcroît, vive et soutenue, à quel point Le Diable au corps peut être considéré comme l'une des «lignes de front» françaises, voire chauvines, de cette véritable "guerre culturelle", tout autant idéologique que financière et industrielle. C'est à cette aune également qu'il faut comprendre l'opposition virulente qu'exprimera la Nouvelle Vague envers la "Qualité française » (on y reviendra).

7 Alain Boillat établit ensuite magistralement comment le flash-back se construit en signature de l'auteur, de l'adaptation littéraire et d'une certaine conception de la "Qualité française ", en dépliant méticuleusement les significations de toutes les pièces d'un dossier génétique profus, celui d'En cas de malheur (1957-1958). Le texte du roman de Simenon, les versions du scénario - et en particulier les enchâssements et analepses, et leur progressive suppression -, les cadrages de certains plans, les dialogues, la musique, la logique du vedettariat de Gabin et Bardot, les discussions du cinéaste et des scénaristes à travers leur correspondance ou des notes sur des feuilles volantes : tout cela est mis à contribution pour formuler l'esquisse d'une histoire du flash-back au cinéma. Une même volonté d'articuler la logique formelle, les logiques de création 
collective et les témoignages des conditions de production anime l'article de Jeanne Rohner sur Occupe-toi d'Amélie!, adaptation d'une pièce de Feydeau. Elle montre notamment comment les prouesses de réalisation (décors, jeux d'acteurs, tours de cirque) veulent affirmer une singularité d'auteur dans le contexte, déjà vu par S. Leahy, de la compétition internationale, mais aussi dans celui, plus local, de la notoriété franco-française et de la rivalité avec Henri-Georges Clouzot (qui réalise lui aussi une adaptation de Feydeau). C'est la mise en abyme, cette fois, qui est la figure recherchée : croquis, correspondance et versions du scénario convergent pour afficher le choix d'une exhibition spécifiquement filmique d'un dispositif théâtral mis à profit pour favoriser la métalepse (jusque dans le dernier regard caméra). Il s'agit ainsi bien de " théâtre à l'écran ", et non de théâtre filmé.

8 Dans les deux articles suivants, Adrien Gaillard et Laure Cordonnier étudient le corpus stendhalien des archives Autant-Lara. L'écrivain justifie, on s'en souvient, le projet de recherche tout entier, initialement destiné à comprendre les adaptations de Stendhal. On approche ici une véritable figure obsédante, évoquée par la couverture du livre (une toile d'Autant-Lara lui-même, donnée à Freddy Buache, et commentée dans l'introduction du collectif), dont la stature gouverne les relations entre le cinéaste et ses scénaristes. A. Gaillard montre que la littérarité s'invite dans le processus d'écriture des documents de réalisation du Rouge et le Noir (1954) ; L. Cordonnier fait de l'obsession stendhalienne, au-delà des collaborations d'Autant-Lara, la marque de cet "esprit français » dans la carrière du réalisateur, depuis les années 1950 jusqu'à ce «film sans images " que sera l'adaptation de La Chartreuse de Parme, qui ne sera pas réalisée (et conduira au procès - perdu - et au pamphlet Télémafia, en 1981). En dépit des pièces reproduisant les documents de projet ou les lettres envoyées aux producteurs, on a le sentiment d'un survol de ces trente ans de défis finalement perdus par Autant-Lara. L'article détaille les ressorts d'une " philosophie stendhalienne » telle que comprise par le cinéaste et telle qu'elle peut être comprise dans l'œuvre de l'écrivain, mais passe rapidement sur l'attaque de Truffaut en 1954, le refus de la Commission d'avances sur recettes de 1971, l'échec du « projet Chartreuse » qui suit. Le positionnement politique d'Autant-Lara, en particulier, aurait pu s'y trouver éclairé, du chauvinisme des temps de la « Qualité française » à la diatribe exaspérée des années 1980. Le choix de faire don de ses archives à la Cinémathèque suisse fut, dit-on, dicté par ce ressentiment envers la France ; ce fut amplement rappelé lors du dernier et plus grave éclat d'Autant-Lara, en 1989, où la provocation antisémite le disputait à la politique abconse ${ }^{3}$, et qui relégua l'artiste jusqu'à ce projet de l'université de Lausanne. Si l'étude n'est jamais illégitime, on peut en attendre qu'elle pose à nouveaux frais les questions les plus aiguës, autant qu'à approfondir les archives méconnues.

9 L'expression " film sans images ", pour décrire le projet " Chartreuse », est emprunté à Jean-Louis Jeannelle. Mireille Brangé et lui signent les deux articles d'ouverture de ce collectif, ainsi que la direction du colloque et du collectif suivant, Films à lire, tiré du colloque «jumeau» de celui de Lausanne. L'ouverture est de taille, puisqu'il ne s'agit plus ni du fonds Autant-Lara, ni des adaptations de Stendhal; le fil conducteur est malgré tout parfaitement visible, puisqu'il s'agit toujours des "Discours du scénario ", chez Antonin Artaud ou chez les sept scénaristes des adaptations envisagées pour $L a$ Condition humaine de Malraux. Après avoir rappelé comme l'exercice intéresse peu les littéraires, à quelques exceptions près, restées sans suite ${ }^{4}, \mathrm{M}$. Brangé prend le cas d'Artaud comme exemple de ce que l'on gagnerait pourtant à intégrer les scénarios à l'œuvre d'un écrivain. La voie peut sembler étroite, car il ne s'agit ni de les lire comme 
des brouillons (avant-textes de films), ni de « les rabattre sur un prétendu caractère littéraire" (p.306). Parce qu'il comporte toutes sortes de cas différents (textes transitoires, vers le film, aussi bien que textes n'ayant débouché sur rien, parce que ce fut impossible ou parce que ce ne fut pas sérieusement envisagé), le cas d'Artaud laisse entrevoir une lecture quasi derridienne du scénario comme débordement, ou périphérie productive. J.-L. Jeannelle, quant à lui, défend une lecture de l'adaptabilité plus encore que de l'adaptation, pour réinscrire les «scénarios inadaptés » (selon son astucieuse syllepse) dans une histoire élargie et approfondie du cinéma : au-delà d'une investigation auteuriste qui voit le scénario inadapté comme une trace dans une carrière mesurée en termes de films réalisés, il s'agit de développer une compréhension du cinéma comme expérience globale et continue, des premiers documents écrits aux variantes des scénarios, «champ de forces en continuelle évolution, où se croisent intérêt littéraire, réputation de son auteur, adéquation de l'œuvre à l'aura d'une star, enjeux politiques et idéologiques, ou même, parmi de multiples facteurs, opportunités diplomatiques » (p. 330) - ainsi de la relance des projets d'adapter Malraux, après que la Chine a réintégré le concert des nations après 1970.

Cette approche culturelle globale offre des perspectives extrêmement suggestives sur les cultures médiatiques, les relations entre littérature et cinéma et les enquêtes de génétique des objets culturels. Déjà fortement élargie par l'intégration des phénomènes de création collective et intermédiatique, où le dossier génétique classique s'enrichit de sources neuves, cette discipline a tout à gagner à une revisitation des pensées de l'avant-texte ; la revue Genesis en a diffusé régulièrement les options, par exemple dans un dossier sur l'après-texte $\left(n^{\circ} 44,2017\right)$.

\section{NOTES}

1. Adrien Gaillard et Julien Meyer, «Jean Aurenche, Pierre Bost et Claude Autant-Lara, auteurs de Douce. Genèse d'une pratique scénaristique », Genesis [En ligne], 41 | 2015, mis en ligne le 20 avril 2017, consulté le 14 mars 2020. URL : http://journals.openedition.org/genesis/1533 ; DOI : https://doi.org/10.4000/genesis.1533.

2. Publié à titre posthume, d'où les guillemets pour le mentionner ici.

3. On évoque ici le ralliement d'Autant-Lara au Front National, jusqu'à être élu au Parlement européen, où il fit scandale en regrettant que Simone Veil ait survécu à la Shoah. Le Monde rapporte qu'il aurait confié à Freddy Buache des visées de gauche, mais stratégiquement dissimulées.

4. Son état de l'art va de la tentative de considérer le scénario comme un genre littéraire (Institut de Littérature Cinématographique Comparée, université Libre de Bruxelles dans les années 1980) au séminaire « Genèses cinématographiques » de l'ENS et de l'ITEM et au travail de J.-L. Jeannelle. 